\title{
Civilisations
}

Revue internationale d'anthropologie et de sciences

humaines

$50 \mid 2002$

Itinéraires belges aux Amériques

\section{Les victimes du sacrifice humain aztèque}

\section{Michel Graulich}

\section{OpenEdition}

Journals

Édition électronique

URL : http://journals.openedition.org/civilisations/3401

DOI : $10.4000 /$ civilisations.3401

ISSN : 2032-0442

\section{Éditeur}

Institut de sociologie de l'Université Libre de Bruxelles

\section{Édition imprimée}

Date de publication : 1 décembre 2002

Pagination : $91-114$

ISBN : 2-87263-180-1

ISSN : 0009-8140

\section{Référence électronique}

Michel Graulich, «Les victimes du sacrifice humain aztèque », Civilisations [En ligne], 50 | 2002, mis en ligne le 01 décembre 2004, consulté le 19 avril 2019. URL : http://journals.openedition.org/ civilisations/3401; DOI : 10.4000/civilisations.3401

\section{(C) Tous droits réservés}




\section{LES VICTIMES DU SACRIFICE HUMAIN AZTEQUE}

\section{MICHEL GRAULICH}

On sait que les victimes du sacrifice humain aztèque étaient très nombreuses. Il fut un temps, sans doute, où c'était surtout des esclaves, comme cela resta le cas chez les Mayas. Mais la forte expansion de l'empire aztèque fit que rapidement, ce furent les guerriers ennemis capturés qui fournirent l'immense majorité des victimes, suivis par les esclaves, les enfants, les condamnés à mort, des personnes anormales: albinos, nains, bossus, contrefaits, macrocéphales, tous sacrifiables d'office, des personnes libres ordinaires, volontaires (comme des prostituées ou des musiciens) ou non, des étrangers de passage et enfin, dans certains cas précis, des princesses de la cité.

Comme ailleurs en Amérique (Helfrich 1973; chez les Nicaraos du Nicaragua: Oviedo 1.40 c.1, 1959, 4:373; Martyr, 6e déc. 1.6, 1965, 2:571), à l'apogée de l'empire, les principales catégories étaient donc les guerriers et les esclaves. Elles correspondent aux deux victimes prototypiques du sacrifice humain dans le mythe dit de la création du soleil et de la lune à Teotihuacan: Nanahuatl, qui est revêtu des atours typiques des guerriers immolés et Tecciztecatl, de ceux des esclaves baignés (Sahagún VII c.5). L'imprécision terminologique des sources ne permet pas toujours de bien les distinguer: le mot (malli) désignant le captif de guerre est employé aussi bien pour le combattant destiné au sacrifice que pour les femmes et les enfants réduits en esclavage (Sahagún II c.20, 1950-81, 2:47; Anales de Cuauhtitlan:37, 59,60...). D'autre part, les guerriers capturés sont parfois appelés esclaves (Sahagún II App., XII c.34; 1950-81 :2:204, 12:95).

La mission des Mexicas est en principe de faire la guerre pour nourrir ciel et terre et pour faire marcher la "machine mondiale". Mais c'est là un discours particulier, propre essentiellement aux nobles, semble-t-il. En fait, il faut faire la distinction entre guerre fleurie et guerre tout court. Dans les deux, on faisait des prisonniers qui étaient sacrifiés. Mais la guerre ordinaire poursuivait avant tout les objectifs habituels d'une guerre. Il n'était pas question d'attaquer une cité sous prétexte de vouloir nourrir le soleil la terre: il fallait des motivations légitimes, telles que le massacre de marchands ou d'ambassadeurs, le refus de commercer, etc. Le roi doit soumettre ses motifs à une assemblée de vassaux, de guerriers distingués et de citoyens de la cité, qui donnent ou refusent leur approbation. En cas de refus, le roi peut reposer la question et, 
après un troisième refus, passer outre et procéder à la déclaration de guerre ou, le cas échéant, à une attaque surprise (Motolinia Memoriales 1.2 c12, 1970:157-158, suivi par Mendieta II, cap. 26, 1:144). ${ }^{1}$ La guerre fleurie (xochiyaoyotl) en revanche a théoriquement pour seul but la capture de prisonniers à sacrifier. Des batailles de ce nom, modérément sanglantes paraît-il, avaient lieu depuis longtemps (Chimalpahin 1965:152; 1997:66-67; Anales de Cuauhtitlan :27, 1945:32), mais la véritable guerre fleurie opposant la Triple Alliance de Mexico, Texcoco et Tlacopan à des cités de la vallée voisine de Puebla, principalement Tlaxcala, Cholula et Huexotzinco, n'aurait été instituée qu'à l'occasion de la grande famine du milieu du $X V^{e} s$., famine qui fut interprétée comme un châtiment des dieux insuffisamment alimentés. Les armées des deux camps s'opposaient régulièrement sur un champ de bataille précis et capturaient le plus possible de prisonniers pour les immoler ensuite au cours des grandes fêtes des vingtaines, garantissant ainsi aux dieux un garde-manger bien fourni. ${ }^{2}$

Deux types de guerres différents selon leurs objectifs, donc, mais deux guerres ritualisées, auxquelles une bonne partie de la population était invitée à s'associer. Les femmes mariées, les jeunes filles, les recluses des couvents se mettaient à jeûner et s'abstenaient de se laver tandis que les prêtres s'extrayaient en outre du sang tous les quatre jours. Le matin, avant le lever de Vénus, les épouses et soeurs des guerriers en campagne allaient dans de petits oratoires où pendaient les mantes des absents et les os des guerriers qu'ils avaient immolés, et elles encensaient et imploraient les dieux et les os de leur donner la victoire. Elles leur donnaient à déjeûner, notamment de grandes tortillas blanches papalotlaxcalli, et les assuraient que leurs parents n'étaient pas partis au travail comme d'habitude, pour gagner leur vie, mais uniquement (!) pour le service des dieux. Le roi jeûnait plus fort que les autres jusqu'au retour de l'armée (Tezozomoc c.70, 1878:539; Durán c.46, 1967 :2:358-359; Pomar 1986:68-69).

La campagne était menée par les "seigneurs du soleil". Les prêtres précédaient les armées d'un jour, portant sur le dos les images des divinités. Avant la bataille, presque toujours en rase campagne, ils s'activaient à allumer $\mathrm{du}$ feu (avec les bâtonnets), puis sonnaient les conques. Dès que le feu avait pris, ils donnaient le signal de l'attaque en poussant des cris. Le ou les premiers ennemis capturés étaient immédiatement immolés devant les images divines (Sahagún VIII c.17 §1; RG d'Ixtepexi, zapotèque; d'Alauiztlan, chontal; d'Ichcateopan, Instlauaca et Tecomastlahuala, mixtèques; Graulich 1994:128). Habituellement, dès le premier choc, une des armées cédait et prenait la fuite. C'est surtout alors, pendant le sauvequi-peut, qu'avait lieu la capture de prisonniers. Les vaincus se soumettent, éventuellement en obligeant leur roi à capituler, voire en le tuant. Habituellement, la chasse à l'homme se prolonge jusque dans la cité ennemie. Parfois, les Aztèques massacrent tout le monde après quelques sacrifices sur place, ou ils tuent quiconque a plus de huit ans, ou encore ils ne laissent en vie, pour les emmener captifs, qu'un jeune homme sur deux, ou les jeunes garçons et filles. Dans d'autres cas, le mot d'ordre est de capturer tout le monde (Motolinia Mem. II c.13-14, 1970:158-159, Mendieta 1945 :1:143-144; Tezozomoc 1878:359, 422-423, 430-431, 342, 344-345; Durán 1967:2. 208, 229, 269, 359).

Quand un guerrier capture un vaincu, il dit: "il est comme mon fils chéri" et l'autre répond: "il est mon père chéri" (ca notatzin: Sahagún II c.21, p.54). La 
capture n'étant pas toujours facile, parfois on coupe les jarrets de ceux qui se débattent. Plus souvent, on s'y met à plusieurs, au maximum six, pour neutraliser un ennemi; par la suite, pour le banquet cannibale consécutif au sacrifice, le corps sera réparti entre les six dans l'ordre où ils ont mis la main sur leur victime. ${ }^{3}$ Si plusieurs guerriers réclament un même captif et qu'il n'y a pas de témoins, c'est un "seigneur du soleil" qui départage, éventuellement après avoir entendu le témoignage du prisonnier. En cas de doute persistant, on peut décider de le remettre au temple Huitzcalco (du calpulli de Coatlan) ou au temple de calpulli (sans précision). Tout tromperie en la matière, comme de s'approprier le captif d'un autre ou de donner son propre prisonnier à autrui est puni de mort (Sahagún 1.8 c.17 \$1, 1969, 2:317; Motolinia Mem. II c.13-14, 1970: 160; Mendieta II c. 27, 1945, 1:144; Pomar), le coupable fût-il fils de roi (Ixtlilxochitl, Historia Chichimeca c.67, 1975-1976, $2: 170)$.

Cette rigueur extrême dans l'attribution précise des victimes s'explique autant par l'importance religieuse de la capture de victimes que par son rôle de moteur social. L'avancement dans la hiérarchie militaire était en effet conditionné, du moins en grande partie, par le nombre de captifs qu'on avait fait immoler. Plusieurs sources plus ou moins concordantes décrivent en détail le processus (Sahagún VIII c.21, 1950-1981, 8:73-75 p.ex.; Cervantes de Salazar I c.22, 1985: 43-44). A partir de l'âge de dix ans, le petit garçon, jusqu'alors rasé, se laisse pousser une mèche sur l'arrière du crâne, mèche qu'on lui rase lorsqu'il a fait un premier prisonnier avec l'aide d'autres personnes. A partir de ce moment, il se laisse pousser une mèche sur le côté droit, jusque sous l'oreille. Elle lui vaudra des quolibets s'il revient deux ou trois fois de la guerre sans captif. Celui qui parvient à faire seul un prisonnier devient un "guide de jeunes gens" que le roi lui-même récompense et revêt de vêtements décorés (Sahaginn 1950-1981: 8:75s.). Les captures suivantes lui vaudront des titres supérieurs et le droit de porter des insignes plus importants. A partir de quatre, il devient "guerrier chef chevronné" (tequihua) et peut prendre place sur la natte de la maison des aigles (cuauhcalli), où se réunissent les grands guerriers. Par la suite, s'il capture encore des Huaxtèques ou d'autres Barbares, il stagne, mais s'il fait des prisonniers de la vallée de Tlaxcala en guerre fleurie il devient huey tiacauh, cuauhyacatl et reçoit du roi de riches atours et insignes. Pour les nobles, des captures dans ces guerres confirment leur noblesse et leur qualité d'aigles-jaguars; elle leur permet de gouverner des cités et de devenir des commensaux du roi. D'autres titres prestigieux étaient ceux de cuachic, guerrier d'arrière-garde et renfort ultime qui jamais ne recule, et d'Otomi ${ }^{4}$

La victoire obtenue, un messager est dépêché chez le roi pour lui annoncer la prise de captifs, puis il est retenu jusqu'à plus ample informé. Sur place, après la prise de la cité, on procède au comptage des captifs obtenus par les différents alliés et des guerriers chevronnés vont faire rapport définitif au roi, en précisant notamment le nombre de nobles qui ont mérité des honneurs pour leur conduite. (Sahagún 1950-1981: 8: 72-73). Ensuite on se met en route avec les prisonniers, dont beaucoup mouraient en chemin. Les Huaxtèques étaient retenus par une corde passée dans le trou qu'ils se percent dans le nez. Aux jeunes qui n'ont pas le nez percé, on met colliers de bois ou on leur entrave bras et pieds. Ceux de Miahuatlan décourageaient toute velléité de fuite en atta- 
chant la corde de leur arc au membre viril du captif. (Durán c. 42, 1967, 2: 327, 330). Le retour à Mexico donnait lieu à une entrée triomphale remarquable. Les anciens guerriers prêtres et les dignitaires de tout rang des temples attendent les prisonniers à l'entrée de la ville, rangés en ordre d'importance, avec leurs atours spécifiques. Ils encensent les "victimes des dieux", leur donnent un pain sacré enfilé sur des cordes et leur disent: "Soyez les très bienvenus à cette cour de Mexico Tenochtitlan, dans la mare où chanta l'aigle et siffla le serpent, où volent les oiseaux, où jaillit l'eau bleue pour se mêler à l'eau rouge $[. .$.$] , où le$ dieu Huitzilopochtli a son pouvoir et sa juridiction. Et ne croyez pas qu'il vous a conduits ici par hasard, ni à chercher votre vie, mais pour que vous mouriez pour lui et offriez au couteau votre poitrine et votre gorge ${ }^{5}$. Et c'est pour cela qu'il vous a été donner de jouir de cette insigne cité, car si ce n'était pour mourir, jamais on ne vous en ouvrirait las portes pour y entrer. Soyez les très bienvenus et ce qui doit vous consoler, c'est que vous êtes ici non par quelque acte femelle et infâme, mais pour des faits d'hommes, pour mourir ici et pour laisser de vous mémoire perpétuelle." Puis on leur donne à boire le pulque divin (teooctli), les assimilant ainsi aux Mimixcoas, les guerriers sacrificiels prototypiques ivres de pulque.

En un premier temps, on s'adresse donc aux prisonniers comme à des ennemis et on met d'emblée les choses au point : ils sont vaincus et leur sort est inéluctable, mais glorieux. En même temps, on leur souhaite la bienvenue, car ils seront bientôt "chez eux" et leur mort les transformera en Mimixcoas et en compagnons du Soleil. On les encense comme des entités sacrées, peut-être aussi pour les purifier en tant que future offrande aux dieux.

Après avoir présenté les victimes à la population, aux prêtres et en particulier aux guerriers qui, frustrés par l'âge ou leurs devoirs, n'ont pu participer à la chasse à l'homme, on les conduit devant le destinataire premier du sacrifice, Huitzilopochtli. ${ }^{6}$ Ils doivent passer en rang au pied de l'idole, dans son temple, en faisant un profond salut, puis, après avoir visité différents endroits qui joueront un rôle lors de leur mise à mort - le cuauhxicalli, le temalacatl, le tzompantli -, ils vont faire de même devant le souverain dans son palais. Celui-ci, qui est comme "seconde personne du dieu [...] et qu'ils adoraient comme des dieux", leur fait donner des vêtements et de la nourriture, voire même des fleurs et des cigares. Le "serpent femelle" ou cihuacoatl, seconde personne du roi, les appelle frères et dit qu'ils sont chez eux, dans leur maison. Il importe en effet d'intégrer les captifs dans le groupe des Mexicas, de faire en sorte qu'ils soient "chez eux". Parfois, ils reçoivent peut-être des filles de joie qui égaient leurs derniers jours et qui sont ainsi, pour un temps, comme des épouses mexicaines de ces étrangers (C.del Castillo 7r,1991:128-9). Nous connaissons le cas d'un captif de marque qui vécut longtemps en liberté dans la ville avant d'être immolé (Graulich 2000a): la victime devient membre de la cité.

Après des danses, dont on ne sait trop si elles sont exécutées par les prisonniers ou leurs vainqueurs ou par les uns et les autres, les seigneurs vaincus viennent faire soumission devant Huitzilopochtli et le devant le roi, en s'extrayant du sang - signe d'humiliation - et en se disant dorénavant serviteurs du dieu. Ils entendent alors les conditions qui leur sont imposées. (Durán c.17, 21, 
1967, 2:159-162, 188) Ensuite, les captifs sont confiés aux calpixque en tant que "grâce du soleil-seigneur de la terre" et fils et vassaux du soleil" (Durán c.21, 1967, 2: 182,188; Tezozomoc c. $29,62,1878: 317,469,530$ ) .

Comment expliquer l'intégration-assimilation des prisonniers, dont l'exemple extrême bien connu est celui des Tupinambas du Brésil (Métraux 1967; Combès 1992)? Par le fait que l'autre est différent, donc moindre, et qu'il ne devient une offrande digne qu'à partir du moment où il est acceptable, intégré ? Ou faut-il évoquer René Girard (1972) et sa théorie du sacrificelynchage ? Selon lui, on ne peut prendre la victime à l'intérieur du groupe, car on risque de provoquer le déchaînement de violence de la vengeance; mais, d'autre part, l'effet apaisant que doit apporter au groupe le sacrifice ne se produit que si la victime en fait partie. Pour concilier ces impératifs, on la choisit marginale (membre du groupe sans l'être tout à fait, un enfant par exemple) ou étrangère, mais alors on l'intègre, on fait comme si la victime appartenait quand même au groupe.

Qu'il y ait dans le sacrifice aztèque une volonté consciente ou non de canaliser la violence interne est possible, mais difficile à démontrer. La composition polyethnique des cités, leur fréquent manque de cohésion, montrent que les risques de violence et de conflits internes n'étaient pas illusoires. Á l'intérieur même de l'île de Mexico, les Mexicas étaient divisés en deux cités dont l'une a fini par asservir l'autre. Á Tenochtitlan, il y avait les habitants les plus anciens, parmi lesquels les Amantèques et les marchands, les calpullis mexicas mais aussi nombre de nouveaux venus, dont les derniers, temporaires, semblent avoir été les Huexotzincas, peu avant la Conquête. D'autres cités de l'époque, comme Texcoco, Cholula, Huexotzinco, etc., abritaient également des populations disparates et notamment, à Cholula par exemple, des Mexicas qui avaient, comme de juste, leur propres intérêts qui ne coïncidaient pas nécessairement avec ceux de leurs hôtes. Les occasions de conflits étaient donc nombreuses et cette spécificité peut avoir été une des causes de l'inflation sacrificielle. Mais, répétons-le, on ne voit pas comment on pourrait démontrer que le sacrifice humain tendait aussi à éviter la violence interne. Ce qui est sûr en revanche, c'est que la théorie aztèque du sacrifice rendait l'intégration de la victime et sa proximité au sacrifiant (ici, en premier lieu, celui qui avait fait le prisonnier) indispensable: le sacrifiant mourait en effet symboliquement à travers sa victime qui le représentait.

Les captifs sont donc confiés aux intendants qui les répartissent dans les maisons de quartier, où ils sont mis dans de grandes cages de bois (Durán c.21, 22, 1967:2:182, 188, RG d'Amula Ameca; Mendieta 1945, 1:151; des cages sont figurées dans Sahagún CF II f.58v, VIII f.27r; la Mapa Quinatzin, etc.). Des personnes désignées les gardent, allant jusqu'à dormir sur ces cages (RG de Metztitlan); si elles doivent sortir pour des besoins naturels, on les retient par une corde passée autour de la taille (Sahagún II c.37). Plusieurs sources s'accordent sur le fait que les prisonniers étaient bien nourris (Sahagún VIII c.14 $\S 8$ ) en vue de leur consommation prochaine (Durán 1967, 2:169, 188; Gómara 1965, 2:31; RG d'Amula Ameca ; Bernal Díaz 1947, 70, 78); par contre, Mendieta (1945, 1:151) affirme que les captifs, mal nourris, devenaient vite 
maigres et jaunes. Probablement cela variait-il selon le nombre de captifs, la période de l'année, la cité intéressée, etc.

Si un prisonnier parvient à s'échapper, le quartier auquel appartient le gardien coupable doit payer en dédommagement un jeune esclave, une rondache et une charge étoffes (Motolinia Mem. II c.13, 1970: 160; Mendieta II c. 27, 1945,1:144), mais on parle aussi de mise à mort du responsable (Durán c.19, 1967, 2: 169). On donnait évidemment la chasse aux fugitifs et selon la relation géographique de Miahuatlan, si on le rattrapait, il était immédiatement mis en pièces. L'évadé de basse condition qui rentrait chez lui était bien reçu et recevait même des étoffes, tandis que s'il était un personnage important, il était sacrifié pour avoir fait honte à sa cité. (Motolinia Mem. 1970: 159-160; Sahagún VIII c.17, 21, 19501981: 8: 53, 75; selon Ixtlilxóchitl 1975-1977, 2:102, le noble est pendu). A Teotihuacan, le prisonnier de guerre mené au sacrifice qui parvenait à fuir, à escalader le temple et à passer derrière la statue du dieu échappait au sacrifice (RG San Juan Teotihuacan 1986:237)

En général, dans les grandes cités de la Triple Alliance et du val de Tlaxcala, on ne relâchait jamais un prisonnier de guerre, surtout s'il était de haut rang (Motolinia, Mem. 1970:160), sauf, paraît-il, pour persuader un ennemi de se soumettre. Dans ce cas, on libérait un grand seigneur qui retournait chez lui pour y rapporter l'effroyable immolation de ses concitoyens (Cervantes de Salazar c.22, 1985:42). Une autre exception concernerait le vaillant qui parvenait à vaincre une seigneur mexica au cours du sacrifice dit "gladiatoire". Paré de la peau du vaincu et de son coeur porté en collier, il était conduit devant le roi qui le nommait capitaine d'une province reculée de l'empire. La source qui rapporte cela, le codex Tudela (fol.12v), n'est cependant pas des plus sûres et donne une version passablement singulière du "sacrificio gladiatorio".

Plusieurs sources affirment que les guerriers capturés étaient tous mis à mort. Les renseignements concernent des cités importantes comme Mexico bien sûr (Durán c.20, 21, 1967, 2: 181-182, 186; Motolinia 1970: 160; Mendieta II c.16, c27; Ixtlilxóchitl 1975-197, 2:145), mais aussi Metztitlan (RG) et les cités qui lui faisaient la guerre. On peut supposer qu'il en allait de même à Tepeucila, dans la Mixteca, où on précise qu'on ne faisait la guerre que pour attraper des victimes à immoler (RG de Tilantongo). Pour d'autres cités, comme Coatlan (RG), près de Miahuatlan, on est confronté à l'ambiguité de la terminologie employée. On y sacrifiait, nous dit-on, "la plupart" des prisonniers, mais le texte poursuit en précisant que les hommes étaient immolés au dieu 7 Lapin et les femmes à 3 Cerf. Ici, visiblement, les captifs de guerre n'incluent pas que les belligérants, étant donné que, normalement, les femmes ne participaient pas au combat, sauf parfois en toute dernière extrémité.

Dans d'autres régions ou royaumes en revanche, on n'immolait pas toujours tous les guerriers prisonniers. Chez les Quichés, seuls les principaux, le seigneur et ses frères, étaient tués et mangés, pour semer l'épouvante (Las Casas 1967, 2: 503-4; Torquemada 1969, 2: 388 suit Las Casas; voir aussi Carmack 1976: 269-71). Chez les Mayas, les captifs de basse extraction étaient réduits en esclavage et les seigneurs sacrifiés, quoique parfois ils pussent se racheter. (Gaspar Antonio Chi, dans Tozzer 1941: 230-32; Bosch García 1944: 95). A Miahuatlan, "de ceux qu'ils prenaient 
en guerre, beaucoup étaient réduits en esclavage", mais on ignore à nouveau si cela concerne aussi les combattants prisonniers (RG Miahuatlan et Tecuicuilco Atepec Coquiapa Xaltianguez). A Tetela (RG) et alentours, "ils se battaient pour faire des prisonniers dont ils tuaient ceux qu'ils jugeaient bon", ou ils les livraient à leur seigneur qui en faisait ce qu'il voulait, comme de les installer sur des mâts et les faire percer de flèches. A Chimalhuacan, non loin de Coatepec (RG), vers 1400 , le roi Tezcapoctzin captura le roi de Xiuhtepec qui paya tribut pour se libérer. Temazcaltepec ( $R G$ ) faisait la guerre contre les Tarasques et les prisonniers étaient sacrifiés ou réduits en esclavage.

Cette situation, où tous les prisonniers n'étaient pas sacrifiés, était fort répandue et peut donc être supposée plus ancienne que l'immolation générale. Certains témoignages indiquent de surcroît qu'à leurs débuts et jusqu'à une date assez tardive, les Mexicas également ne sacrifiaient qu'un nombre limité de guerriers. Ixtlilxochitl (1975-77, 1: 330) par exemple relate qu'en 1359, les Tépanèques capturèrent des Acolhuas dont eux-mêmes et leurs alliés mexicas ne sacrifièrent que les vaillants, les autres étant vendus comme esclaves. Sous Axayacatl, des prisonniers de guerre matlatzincas servirent à repeupler la cité de Xalatlauhco, et on peut supposer que ce n'étaient pas seulement des femmes, des enfants et des vieillards (Ixtlilxóchitl 1975-77, 2:144; voir aussi Durán c.28, 1967, 2: 229: les esclaves appartenaient à ceux qui les avaient capturés mais parfois le roi les prenait pour les immoler, "mais il en donnait le double de richesses de ce qu'ils valaient"). L'immolation générale semble donc être un développement tardif à mettre en rapport avec la constante croissance démographique de l'époque ainsi qu'avec la grande extension de l'empire aztèque et l'afflux de captifs dans les cités puissantes. Cette évolution va de pair avec une manière de "démocratisation" du sacrifice humain et du cannibalisme, qui deviennent accessibles même aux guerriers issus du peuple.

Non seulement les cités ne sacrifient pas toujours tous leurs captifs,

mais souvent, si elles ne sont pas indépendantes, il leur est interdit de le faire parce qu'elles doivent de livrer une partie de leurs captifs en tribut à la cité dont elles dépendent, et ce selon des modalités qui peuvent varier. Tel est le cas de Tepeaca qui, vaincu par Montezuma I doit fournir tous les 80 jours des captifs de guerre à s. à Mexico (Durán c.18, 1967, 2:158), ou de Tlatelolco, qui, lors de chaque campagne, doit livrer des prisonniers de guerre pour Huitzilopochtli (Tezozomoc c.46, 1878: 397). Les Tépoztèques de Citlalatomaua, dans le Guerrero méridional, ne sont pas cannibales mais font la guerre pour livrer des esclaves à manger à Montezuma. (RG de Citlalatomaua; aussi RG de Zumpango). Lorsque ceux d'Acapetlaguaya (RG) capturent une "personne principale", ils l'envoyent au roi à Mexico pour qu'il l'immole. Ceux d'Ahuatlan (RG), près de Puebla, "ne paient pas tribut" à Mexico mais participent à la guerre fleurie et conduisent leurs captifs au sacrifice à Mexico. Les Zapotèques d'Ocelotepec (RG; voir aussi Soustelle 1955:102) sont toujours en guerre et quand la prise est bonne, ils envoient quelques captifs à Montezuma "en signe de reconnaissance", "comme présent". D'une manière plus générale, Durán (c.43, 1967, 2:334) raconte que lors de l'inauguration de la pyramide principale du Grand Temple sous Ahuitzotl, on attendait des seigneurs invités qu'ils apportent l'habituel tribut d'esclaves "qu'ils 
étaient tenus d'apporter pour le sacrifice lors de telles solennités" et il est précisé plus loin qu'il s'agit de "tous les captifs pris en guerre qu'ils devaient en tribut à la Couronne royale de Mexico". Enfin, on sait qu'à l'occasion de la fête de Macuilxochitl, donc le jour 5 Fleur, "de partout on amenait des captifs, appelés maltequime" (Sahagún I c.14, 1950-81, 1:32).?

J'ai démontré ailleurs que la chasse à l'homme qu'était la guerre était à bien des égards assimilée à une chasse tout court (Graulich 1997). Chez les Quichés, les captifs étaient du reste qualifiés de gibier. Non seulement la proximité homme-animal était très grande, mais, on l'a vu dans les mythes, les hommes ont remplacé les animaux comme victimes. Les sources (Anales de Cuauhtitlan fol.1-3 p.ex.) laissent aussi entendre qu'avant la création du soleil, alors que les peuples nomadisaient, Terre et Feu, qui alors dominaient, se contentaient des prémices de la chasse. Cette offrande autorisait la manducation du reste, de même que seul le sacrifice humain autorisait le cannibalisme. Les captifs entrant à Mexico hurlaient comme des bêtes; une fois sacrifiés, leurs têtes étaient exposées sur le tzompantli où elles devenaient les fruits d'une sorte de verger artificiel qui devait assurer la renaissance des victimes, de même que les rites effectués avec les os du gibier assuraient son retour. La guerre imitait donc la chasse, mais parfois c'était l'inverse, comme lors des fêtes de quecholli, de tititl et d'izcalli (toutes au cours de la saison des pluies, nocturne, présolaire), au cours desquelles avaient lieu de grandes battues au terme desquelles le gibier était sacrifié comme des hommes ou offert au feu (ce qui pouvait également arriver à des guerriers), tandis que les capteurs étaient récompensés comme des guerriers. En quecholli, on allait même jusqu'à assimiler des captifs à des cerfs et à les sacrifier comme tels. A cette égard, la quête du peyote des Huichols actuels est particulièrement intéressante, car, tout en prolongeant la réactualisation précolombienne des pérégrinations vers la Terre promise ou la terre d'origine, elle perpétue aussi la chasse sacrificielle, le peyote étant assimilé au maïs, au cerf et à l'homme. A l'instar du sacrifiant aztèque, le peyotero, grâce au peyote mis à mort et mangé, monte au ciel et voit son dieu en face.

Les guerriers sacrifiés n'étaient pas tous d'égale qualité. Il va de soi que la capture d'un roi, d'un seigneur, d'un noble, d'un haut gradé ou d'un vaillant était plus prestigieuse que celle d'un soldat ordinaire et que ces personnes avaient en elles plus de tonalli, de feu intérieur, de chaleur vitale susceptible de vitaliser les dieux et les hommes (Sahagún IX c.2, 1950-81, 9:3; aussi chez les Mayas p.ex.: Helfrich 1973: 51-70). Certains types de mise à mort leur étaient d'ailleurs réservés, en particulier le "gladiatorio", qui opposait un prisonnier attaché à une meule par une corde passée autour de la taille et pratiquement désarmé à des guerriers aigles ou jaguars pourvus d'épées à tranchants d'obsidienne. On dit que c'est le roi lui-même qui choisissait les victimes dignes de cet honneur après de multiples vérifications (Pomar 1986: 66). Si la victime royale ou princière avait été capturée par un roi ou un seigneur, on conservait sa peau que le vainqueur revêtait parfois ou qu'on bourrait de coton ou de paille et pendait dans le temple ou le palais. (Motolinia Mem. I c.18, II c.14, 1970: 33, 161). 
Autre sacrifice de privilégié, celui que faisaient les Popolocas en l'honneur du "dieu papier" Amateotl, à qui, après la victoire, ils immolaient le "meilleur prisonnier" fait en guise d'action de grâces. Ils trempaient du papier dans le sang du coeur de la victime et le collaient sur l'idole (Histoyre du Méchique 1905: 95).

Il y avait aussi des différences de qualité entre les peuples. Les victimes les plus appréciées étaient celles qui appartenaient à des populations peu éloignées et dès lors pas trop différentes de la Triple Alliance (Motolinia Mem. I c.21, 1970: 35). Les Yopis, les Tarasques du Michoacan ou les Huaxtèques vivaient trop loin. "La chair de ces populations barbares, explique le cihuacoatl Tlacaelel, qui suggéra la guerre fleurie, n'est pas agréable à notre dieu. Il la tient pour du pain bis et dur et pour du pain fade et non relevé car, comme je le dis, ils sont barbares et de langue étrangère". Les Tlaxcaltèques en revanche "sont comme du pain chaud à peine sorti du four, tendre et savoureux". Ce sont là, avec ceux de Huexotzinco, Cholula, Atlixco, Tecoac et Tliliuhquitepec, les victimes préférées de Huitzilopochtli. Les Tlaxcaltèques, les Cholultèques, les Mexicas, les Huexotzincas sont tous d'une même famille, ils appartiennent à une seule et même génération, celle des Chichimèques... (Durán 1967,1: 32; 2.232-235, 417, 449; Muñoz Camargo $1892: 116)^{8}$

L'origine spécifique de certaines victimes pouvait aussi leur conférer une valeur particulière. Ainsi, au mois d'izcalli, consacré au dieu du feu Xiuhtecuhtli, on sacrifiait des victimes appelées Ihuipaneca Temilolca, c'est-àdire, "ceux de la passerelle de plumes, ceux des colonnes de pierre" (López Austin 1965: 96; Garibay 1956: 4: 354). Ihuipan peut signifier aussi "sur la plume" ou "drapeau de plumes". On sait par les Anales de Cuauhtitlan (p.1) qu'une des trois pierres du foyer s'appelait Ihuitl, "Plume"; peut-être y avait-il un rapport avec le nom des victimes qui accompagnaient le dieu du feu, dans lequel cas les "colonnes de pierre" pourraient également faire allusion aux pierres qui "gardaient" le feu. Mais les Ihuipaneca Temilolca nous sont connus d'autre part. Chimalpahin parle fréquemment des "anciens", un peuple qu'il appelle les Eztlapictin Teotenanca Teochichimeca Cuixcoca Temimilolca Ihuipaneca Zacanca, et dont le dieu national n'était autre que Nauhyotecuhtli Xippilli, "Seigneur du Quadruple", Prince de Turquoise", c'est-à-dire Xiuhtecuhtli (Chimalpahin, Memorial Breve f.33r, 1991:50). Sans doute les captifs appartenaient-ils à ce peuple qu'ils représentaient donc et que personnifiait leur dieu tutélaire, le dieu du feu.

Enfin, les futures victimes pouvaient aussi être traitées différemment si elles appartenaient à un roi ou à un seigneur. Dans ce cas, elles entraient dans la ville en tenant des rondaches et des épées ou encore des encensoirs, des cigares allumés et des fleurs, et en chantant le chant de leur pays, en pleurant et en lamentant leur malheur." Si leur vainqueur était un roi, elles étaient transportées en litière et recevaient de riches donc (Tezozomoc c.38, 49, 1878: 360, 410, Mendieta 1945, 1:146).

Il semble bien, enfin, que parfois des femmes pouvaient être sacrifiées de la même manière que, et avec des prisonniers de guerre, pour des raisons qui ne sont pas spécifiées. Il y a à cela des précédents mythiques. D'abord, bien sûr, le mythe de Coatepec où les 400 Huitznahuas et leur soeur aînée Coyolxauhqui s'arment comme des guerriers pour aller tuer leur mère enceinte au sommet de la colline de Coatepec. Mais Huitzilopochtli naît et tue en 
premier lieu Coyolxauhqui, qui devient ainsi la toute première victime de Huitzilopochtli. D'autre part, selon les codex Boturini et Aubin, au début de leurs pérégrinations vers leur terre promise, les Mexicas voient, gisant sur des acacias et des cactées, trois Mimixcoas dont une femme, Chimalman, et Huitzilopochtli leur commanda de les sacrifier en tant que prémices de la guerre sacrée menée pour le nourrir. Là aussi, à en croire le Boturini, la femme fut la première victime. ${ }^{9}$ Toujours pendant ces errances, après la défaite de Chapultepec, les filles du roi Huitzilihuitl réclamèrent la craie et les plumes, c'est-à-dire d'être sacrifiées comme des guerriers, ce qui eut lieu (Anales de Tlatelolco 1948: 36-8). E’nfin, après la prise de Tlatelolco par Axayacatl, la reine de cette cité plaignit les femmes, se demandant si on les mènerait au sacrifice avec les guerriers (Tezozomoc c.63, 1878: 384). Dans ce dernier cas, on a au moins un semblant d'explication puisque, dans la bataille qui s'ensuivit, des femmes nues mais armées firent mine de se battre avec les Mexicas-Tenochcas, tandis que d'autres les bombardèrent de balais, d'instruments de tissage, de lait pressé de leurs seins et d'ordures mélangées à de la terre. Bataille dérisoire, moqueries qui signifiaient que les Tenochcas n'étaient dignes de se battre que contre des femmes, mais participation des femmes à la guerre quand même. D'autre part, l'héroïsme féminin était officiellement reconnu puisque les femmes mortes en premières couches devenaient dans l'au-delà des femmes guerrières compagnes du soleil de l'après-midi.

Dans tous les cas considérés jusqu'à présent, les guerriers sacrifiés sont des ennemis. Il faut toutefois signaler un cas tout à fait exceptionnel où des guerriers mexicas pouvaient être sacrifiés à Mexico. Pendant la fête du mois de panquetzaliztli, une bataille rituelle sanglante opposait des esclaves baignés figurant sans doute Huitzilopochtli à des personnificateurs des demi-frères ennemis du dieu, les 400 Huitznahuas, aidés par des vaillants mexicas. Dans ce combat, les esclaves sacrificiels qui réussissaient à capturer un guerrier l'immolaient sur tambour de bois horizontal en guise d'autel (Sahagún II c.34, 195081, 2: 146).

Les attributs les plus caractéristiques dont on ornait les victimes guerrières étaient les rayures de craie (tizatl) sur le corps et le duvet (ihuitl) collé sur la tête avec la résine d'une sorte de pin (Martínez Cortés 1974: 42). ${ }^{10}$ Cristóbal del Castillo (f.7, 1991: 126-29) présente cette pratique comme typiquement mexica, puisque c'est le dieu Tetzauhteotl même qui, au cours des pérégrinations mexicas, aurait instruit un Huitzilopochtli évhémérisé de la manière de traiter les prisonniers de guerre: "Tercera cosa: a los que harán cautivos los pintarán de blanco, los emplumarán con plumón ligero, los curarán, los atarán por el vientre con un cordel grueso y les colgarán plumas de garza. Los harán comer mucho para engordarlos, y cada vez que se cumpla una veintena los matarán, y para que se celebre la fiesta andarán danzando. $Y$ cuando sea la víspera de su muerte, velarán toda la noche, comerán, danzarán y se emborracharán ; y si acaso alguno quiere acostarse con mujeres, le serán ofrecidas prostitutas, habrá muchísimas mujeres perversas, prostitutas. ${ }^{111}$ Mais en fait, cette origine mexica est illusoire: les figurations de guerriers ornés de craie et de plumes sont très 
répandues et se retrouvent notamment dans les codex du groupe Borgia, où Huitzilopochtli brille par son absence, ou encore dans des codex mixtèques (Nuttall p.4, 20). De plus, dans l'antique mythe pré-aztèque de la création du soleil et de la lune à Teotihuacan, la craie et les plumes ornent Quetzalcoatl, prototype des guerriers héroïques sacrifiés.

Les termes mêmes de tizatl, ihuitl peuvent à eux seuls désigner les victimes guerrières, voire même le guerrier en général, ou encore le sacrifice, comme on l'a vu plus haut, à propos des filles de Huitzilihuitl. ${ }^{12}$ Á ces attributs s'ajoutaient habituellement la bannière et les atours de papier. Les informateurs de Sahagún (Sahagún II 2 c. 29, 1950-81, 2:113) décrivent comme suit les guerriers sacrifiés en xocotl huetzi: "on les a couverts de craie (quintiçavia), d'un pagne de papier blanc (amamaxtli), de sortes étoles blanches (amaneapanalli), d'une chevelure de papier (amatzontli), on leur a emplumé la tête (quinquapotonja), ils ont la lèvre ornée du labret de plumes (imjvitençac), ils ont la bouche peinte en rouge (motenchichiloa), ils ont le pourtour creusé des yeux teint en noir (mjxtentlilcomoloa)".

Il faut souligner encore que tous ces attributs sont ceux de Mixcoatl et des Mimixcoas, prototypes toltèques des guerriers sacrifiés (Durán 1967, 1:73 et pl.13; Codex Telleriano-Remensis f.29v, 38v, 39r; Codex_Magliabechiano p. 42; autres codex: Spranz 1964: 8998;voir aussi Seler 1890: 608, 613-4; 1902-23, 1:264-5; 2:1019; 4:66-7, 72-5, 84-5). Il est tout à fait remarquable que même en panquetzaliztli, au cours de ce mois qui voyait la réactualisation de la victoire de Huitzilopochtli à Coatepec, les multitudes de guerriers sacrifiés étaient toujours affublés comme des Mimixcoas et non des Huitznahuas. Les Mimixcoas étaient les adversaires malheureux de Quetzalcoatl sur la Montagne de Mixcoatl, le Mixcoatepec. Ce mythe du Mixcoatepec servit manifestement de modèle aux Mexicas pour créer leur mythe "national" de la victoire de Huitzilopochtli à Coatepec. J'ai dit ailleurs ma conviction que Mexico-Tenochtitlan, à son origine, était une cité dont la divinité tutélaire était Quetzalcoatl, le vainqueur du Mixcoatepec. Lorsque les Mexicas s'emparèrent du pouvoir dans cette ville, ils mirent Huitzilopochtli à la place de Quetzalcoatl dans les temples et dans les rites. Mais, on le voit, ils n'eurent même pas le temps, ou l'occasion, ou le souci, de modifier complètement les rituels en conséquence (Graulich 1992, 1999: 187-92).

Seler (1902-23, 4: 96-7) voit dans le duvet blanc des nuages, les âmes des morts devenant des messagers de la pluie. Pour Soustelle (1940: 72), il était le symbole de l'heureux destin de la victime, le blanc étant "la couleur des premières lueurs du jour" et donc "du premier pas de l'âme ressuscitée, l'envol vers le ciel du guerrier sacrifié". Pour moi, les plumes et la craie marquaient l'appartenance de la victime au ciel et à la terre. Les plumes sont des éléments aériens; des boules de duvet, Durán (Ritos c.5, 1967, 1:47 et pl. 9; l'auteur parle de "boules de coton" mais l'illustration montre les habituels duvets) disait qu'elles étaient "les vêtements du ciel". La craie, par contre, est terrestre. Le rite consistant à s'humilier en mangeant de la terre s'appelait "goûter la craie" (Sahagún 1958a: 50-1 et Durán_Ritos c.15, 1967, 1:147-8). ${ }^{13}$ Dans le Popol Vuh, c'est pour avoir mangé un oiseau enduit de craie que Cabracan, alourdi, s'effondra et fut enseveli.

On ne sait pas grand-chose sur la signification de cette sorte de loup noir peint autour des yeux et du rouge qui entoure la bouche. Pour ce second 
élément, peut-être y a-t-il un rapport avec le fait que la bouche des idoles destinataires des sacrifices était ointe du sang des victimes. Quant à la peinture noire autour des yeux, elle est aussi appelée "peinture stellaire". Le rapport entre les yeux et les étoiles est bien connu, puisque dans l'iconographie mésoaméricaine, les étoiles sont souvent figurées comme des yeux stylisés. Mais la "peinture stellaire" doit probablement aussi rappeler que, la nuit, les guerriers morts deviennent des astres, comme Quetzalcoatl qui devint le soleil ou Vénus, ou comme les 400 jeunes gens du Popol Vuh - équivalents des 400 Mimixcoas ou des 400 Huitznahuass - qui devinrent les Pléiades (Seler 1902-23, 4: 72-84; Caso 1953: 53)

Les guerriers voués au sacrifice ne représentaient pas seulement les Mimixcoas ou peut-être, parfois, les Huitznahuass. Dans le "gladiatorio", les victimes, qu'on écorchait, étaient Xipe Totec, l'antique dieu vêtu d'une peau d'écorché attesté, avec ses attributs, dès la phase Monte Albán III chez les Zapotèques. C'était une divinité complexe dont on ne sait trop si les victimes le représentaient ou si c'était l'inverse. Quoi qu'il en soit, ses connotations étaient à la fois solaires, lunaires, vénusiennes et en rapport avec le maïs (Graulich 1987). Ses victimes portaient un chapeau conique, une courte jaquette, des ornements et des rubans rouges et blancs (couleurs, aussi, des Mimixcoas) se terminant en queue d'aronde et des triples noeuds en papier. Ils avaient également les bras et la tête couverts de duvet blanc. (Codex Nuttall et Tudela p.ex.).

Les guerriers voués au sacrifice incarnent-ils parfois d'autres divinités, plus individualisées que les Mimixcoas ou Xipe, qui ne sont avant tout, somme toute, que de la nourriture pour dieux? La question est complexe, comme l'illustre le cas de l'ixiptla, le personnificateur, l'image de Tezcatlipoca, sacrifié au mois de toxcatl. Précisons qu'un des aspects de Tezcatlipoca est celui de Yaotl, le Guerrier. A Texcoco, selon notre meilleure source sur cette cité, Pomar (1986: 67), c'était indiscutablement un vaillant captif de Huexotzinco ou Tlaxcala qui l'incarnait. A Mexico, ç'aurait été un esclave baigné d'après Durán (1967, 1:59 ainsi que Tovar 228 et Acosta 270-1, mais ces deux sources dépendent de Durán), mais Sahagún (1950-81, 2:66-8; 1956, 1: 114, 152-3), lui, est moins net: l'ixiptla du dieu était choisi parmi les nombreux ixiptla gardés et entretenus par les intendants (calpixque); il y en avait environ 10, des captifs (mamalti) choisis parmi les p risonniers de guerre. Seulement, on l'a vu, les prisonniers de guerre ne sont pas nécessairement des guerriers: ce sont toutes les personnes, hommes, femmes et enfants capturées lors d'une campagne. Le texte espagnol parle d'ailleurs d'un "mancebo escogido", "mancebo, muy acabado en disposición", choisi pour son aspect physique qui devait être parfait, sans la moindre tache ou marque -ce qui, pour un guerrier, était plutôt rare. ${ }^{14}$

La question est tout aussi douteuse pour le personnificateur du dieu du feu Xiuhtecuhtli en izcalli. Motolinia (I, c.19, 1970: 33) le dit un prisonnier de guerre, "uno de los cautivos en la guerra". Il pourrait bel et bien s'agir d'un guerrier, d'autant plus que dans un autre passage du même chapitre, l'auteur semble (mais pas nécessairement) faire la différence entre esclaves et captifs en parlant de "algunos esclavos y otros cautivos que tenian de guerra". On ne sait malheureusement pas dans quelle cité se déroule l'izcalli décrit par Motolinia. 
Mais il est certain qu'à Mexico et à Tepepulco, le ou les ixiptla du feu étaient des esclaves baignés (Sahagún II c.37-38 et Primeros Memoriales; Codex Tudela f.28; Olivier 1997: 2345).

Enfin, en tlacaxipehualiztli à Tlaxcala, il est question d'un "fils du soleil" immolé lors de l'allumage du feu nouveau à minuit (Motolinia I, c.27, 1970: 42). C'était "uno de los más principales" dont on peut conjecturer que c'était un guerrier, comme lors des grandes fêtes séculaires du feu nouveau (Sahagún 195081, 7:25-28). Seulement, un "fils du soleil" peut être, mais n'est pas nécessairement, un personnificateur de l'astre. Les mêmes réserves concernent le "messager du soleil" sacrifié le jour 4 Mouvement, nom de l'ère actuelle (Durán 1967, 1:106-8). Dans une des ces interprétations audacieuses dont il est coutumier, Durán affirme que son ascension des marches de l'édicule sacrificiel imite la course du soleil et donc, que la victime joue le rôle de l'astre. Mais elle n'en a pas les attributs et est immolée d'abord à la terre, puis au soleil, car elle est d'abord décapitée et ensuite seulement, on lui arrache le coeur. Ajoutons que dans un cas au moins, les guerriers immolés peuvent représenter des cerfs, le gibier par excellence. On sait en effet qu'à l'aube de l'ère actuelle, les Mimixcoas furent massacrés parce que, lorsqu'ils tuaient du gibier, ils n'en faisaient pas d'abord l'offrande à leurs parents, Soleil et Terre. Aussi devinrent-ils désormais euxmêmes la nourriture de ces dieux (Graulich 2000a).

La mort sacrificielle est en effet un châtiment. Les hommes ont négligé leurs créateurs et doivent expier. Qui plus est, ils sont nés sur terre, dans la matière qui les sépare des dieux et les condamne à mourir. "Notre tribut est la mort, que nous avons méritée " (Sahagún VI c.1). Par la mort hérö̈que, acceptée, sur le champ de bataille ou la pierre de sacrifice, le guerrier expie et gagne un au-delà glorieux. C'est pourquoi on dit au jeune homme qui avec l'aide d'autres, fait un premier prisonnier, au travers duquel il mourra symboliquement et expiera, que "Tonatiuh Tlaltecuhtli t'ont lavé la face" (Sahagún VIII c.21, 1950-81, 8: 75 p. ex.). Châtiment salvifique, la mort sacrificielle du guerrier est ressentie à la fois comme une gloire, un honneur, et un malheur. Parmi les catastrophes qu'on annonce à ceux qui sont nés un jour néfaste figure régulièrement le sacrifice. Par exemple, celui qui naît le jour 1 Maison périra d'une mort dangereuse, en guerre ou sur la pierre de sacrifice ou comme esclave baigné, ou bien il commettra un adultère et sera mis à mort ainsi que sa complice, ou il sera vendu comme esclave, ou volera, ou se ruinera au jeu (Sahagún IV c.3; V, c.1). Entendre rugir un jaguar est un mauvais présage qui annonce la mort en guerre (Sahagún V c.1). Celui qui gagne au jeu de balle est "grand adultère, et mourra en guerre ou des mains d'une mari outragé" (Tezozomoc c.2, 1878: 228). Les proverbes et les métaphores aussi sont éloquents. Pour signifier qu'on a bien mis quelqu'un en garde, on dit (Sahagún VI c.43): "je t'ai donné ton petit drapeau que tu devras prendre en mourant, je t'ai donné ton papier sacrificiel". A un coupable dont on accepte de taire la faute, mais dont on exige qu'il s'amende, on dit: "je t'applique de la craie blanche et des plumes, je te donne ta bannière et le papier sacrificiel [...] je t'enfonce dans la terre, je te donne l'eau épineuse, l'eau de douleur...". Perdre tout espoir, c'est "recevoir la bannière, les bandes de papier sacrificielles" (Sullivan 1963; Preuss 1903a: 190-1; 
1903b: 256-7). Enfin, Tezozomoc (c.70, 1878: 516), parle de "pénitent" désigner la victime sacrificielle.

Il reste un dernier et vaste sujet qui ne pourra être qu'effleuré ici, c'est celui du nombre des victimes. Un sujet éminemment sensible, qui souvent fait perdre tout sens critique aux chercheurs, trop prompts à vouloir minimiser à tout prix (Graulich 1991). Il est clair que dans les cités les plus puissantes, les victimes étaient très nombreuses; elles le paraîtront un moins si on considère que nombre d'entre elles, normalement, auraient dû mourir sur le champ de bataille. Il est évident aussi que le nombre de victimes a fortement augmenté à mesure que la puissance grandissante de la Triple Alliance s'appuyait de plus en plus sur la terreur pour assujettir les population. Cette croissance va aussi de pair avec celle de la démographie, surtout entre, mettons, 1450 et 1519.

S'il faut en croire Tezozomoc, sous Montezuma I, les Mexicas se réjouissent fort d'avoir fait 200 prisonniers de Chalco en une bataille (donc, probablement des guerriers). Quelques décennies plus tard, Ahuitzotl ramènerait 44.000 captifs (sans doute de tout ordre) de sa campagne au Guerrero; la région devra être repeuplée par la Triple Alliance. De sa campagne contre Tututepec, Montezuma II et ses alliés ramènent 1350 prisonniers, et une autre fois, 2800, mais une bataille fleurie contre Huexotzinco ferait 10.000 morts. Après la prise de Mexico par Cortés, celui-ci ne peut empêcher ses alliés, notamment tlaxcaltèques, de sacrifier et de manger plus de 15.000 ennemis, chiffre qu'il n'avait pas intérêt à gonfler (Gómara 1975, 2:114).

Les guerres, faut-il le dire, étaient incessantes et les cités soumises devaient souvent livrer des victimes à sacrifier comme tribut. Dès lors, des fêtes au cours desquelles on immole quelques milliers de victimes deviennent assez rapidement chose courante à Mexico. Durán $(1967,2: 415$; aussi 443, 482) affirme que sous Montezuma II, il y avait des jours de deux, trois, cinq ou huit mille sacrifiés à Mexico. Pour l'inauguration du temple de Tlamatzinco et du cuauhxicalli, il y en aurait même eu 12.210. On est loin, bien sûr, des chiffres controversés de l'inauguration du Grand Temple de Mexico en 1487 par Ahuitzotl. La plupart des sources en nahuatl et en espagnol s'accordent sur le chiffre de 80.400 victimes sacrifiées à cette occasion, ce qui paraît énorme et a bien sûr donné lieu à toute une littérature révisionniste, certains allant même jusqu'à proposer le chiffre parfaitement fantaisiste de 320 morts seulement. Il faut dire que la circonstance était exceptionnelle. Les travaux ayant débuté sous Tizoc, celui-ci a d'emblée dû se mettre à stocker les victimes pour l'inauguration. Mais il mourut prématurément et Ahuitzotl lui succéda. Il acheva l'agrandissement de la pyramide dont il fit coïncider l'inauguration avec son intronisation, en vue de laquelle il fit également une campagne. Théoriquement, les victimes de cette vaste opération de terreur ont donc effectivement pu compter des dizaines de milliers de victimes.

Pour la moyenne annuelle de victimes, les estimations anciennes diffèrent. Une lettre du moine évêque Zumarraga mentionnée par Torquemada $(1969,2: 120)$ mentionne 20.000 enfants sacrifiés par an, mais Clavijero (1964: 172) évoque une autre lettre où ce seraient 20.000 personnes par an dans la seule 
ville de Mexico. Gómara (1975, 2: 91, 435) semble faire de 20.000 à 50.000 victimes le total pour le pays tout entier. Il semble s'appuyer sur un propos d'un certain Ollintecuhtli qui, vantant la puissance de Montezuma, aurait dit à Cortés qu'il sacrifiait 20.000 personnes par an. Las Casas (1985: 247-8) en revanche, parfaitement de mauvaise foi, admet tout au plus 10 ou 100 sacrifices par an alors que dans son Apologética, il reprend les données habituelles des autres sources. Dernier élément qui montre le grand nombre de sacrifices, c'est celui concernant les tzompantli ou plates-formes d'exposition des têtes des défunts. Je passe sur le tzompantli imaginaire inventé par Bernal Díaz à Xocotlan, mais à Mexico, selon le conquistador Andrés de Tapia qui, avec un collègue, les aurait comptées, le nombre de têtes s'élevait aux alentours de 136.000 . Ici encore, le total est peut-être excessif, mais il montre bien que les victimes étaient nombreuses. Victimes qui, rappelons-le encore, dans des pays dotés d'armes meilleures et où on n'essayait pas de faire prisonnier pendant et après le combat, seraient mortes sur le champ de bataille.

Outre les guerriers, il y avait toute une série d'autres victimes que je ne puis que survoler. Les esclaves, fort nombreux, provenaient de la guerre, du tribut ou étaient des condamnés, des enfants vendus ou des personnes qui se vendaient. Seuls pouvaient être sacrifiés, semble-t-il, les condamnés, les esclaves pour dettes de jeu qui ne pouvaient se racheter et les esclaves indociles qui avaient été vendus deux ou trois fois (Motolinia 1970: 174; Durán 1:183, 200, 210) . A Mexico du moins, on n'aurait pas sacrifié d'esclaves étrangers (Durán 1:182), quoique selon Cortés (1963: 26; Durán 2: 465), il y aurait eu aussi des esclaves livrés comme tribut. La tendance à sacrifier de préférence des proches, plus semblables et donc meilleurs, a déjà été observée à propos des guerriers et cadre bien avec l'idée que la victime est substitut du sacrifiant. Si celui-ci était l'Etat, il pouvait donc puiser dans les esclaves de tribut, par exemple. Sinon, il fallait acheter au marché, au prix de 30 à 40 étoffes par esclave, selon sa qualité, sa perfection physique, ses aptitudes, notamment à chanter et à danser (Sahagún IX c.10). Avant d'incarner une divinité pendant un nombre de jours variable, la victime était rituellement baignée, selon des modalités variables (parmi lesquelles il ne faut pas inclure, semble-t-il, les bains quotidiens à l'eau chaude destinés à engraisser la victime), de manière à la purifier des souillures de sa condition d'esclave (Durán 1: 64, 181-2). Celle-ci était en effet considérée comme un châtiment, non seulement pour des délits sanctionnés par la loi, mais aussi pour une vie déréglée. Certaines circonstances requéraient des jeunes vierges, parce que les déesses incarnées, ou ce qu'elles représentaient (p.ex., le maïs), l'étaient.

Un troisième groupe de victimes, bien moins nombreux, comprenait des condamnés à mort, une confirmation de plus du fait que la mort sacrificielle est expiatoire. Durán (1:185) dit clairement que le sacrifice était une des façons de châtier les criminels. Une bonne partie des condamnations étaient en rapport direct avec le sacrifice, la guerre et le culte (immolation de guerriers nobles qui, capturés, s'étaient échappés et étaient rentrés chez eux; de gardes qui avaient laissé fuir des captifs; de gens du commun ayant refusé d'assister à des sacrifices humains; de serviteurs ayant laissé s'éteindre le feu domestique lors de la fête du Feu nouveau; d'ennemis de la "guerre fleurie" trouvés en territoire 
adverse; d'émissaires considérés comme traîtres; de voleurs de biens du temple... ${ }^{15}$ ), mais elles concernaient aussi les sorciers et les devins qui se trompaient (Torquemada 2: 386), dans certains cas les adultères et, au Guatemala, les violeurs de vierges (Castañeda Paganini 1959: 33). On sait par les codex (Borbonicus, Telleriano-Remensis...) que des exécutions d'adultères pouvaient avoir lieu en présence de la divinité. On tuait de même des criminels et des guerriers devant le souverain pour le "vivifier, renforcer son tonalli (feu intérieur)" (le texte, ambigu, peut désigner les seuls guerriers) (Sahagún IV c.11, 12). Se pose alors la question du destin dans l'au-delà de ces exécutés: partageaientils celui des sacrifiés? Certaines indications le suggèrent, comme le fait que les parents d'un condamné pour adultère lui faisaient une image de la déesse de l'amour et de la saleté.

Les victimes dont il a été question jusqu'à présent sont soit extérieures à la cité, soit marginales. D'autres marginaux sacrifiables sont les enfants -incomplètement intégrés dans le groupe et dépendant de leurs parents- et les personnes anormales, donc potentiellement dangereuses, ou marquées. Les sacrifices d'enfants, victimes faciles à obtenir, évidentes, étaient très fréquents. Des enfants de rois ou de seigneurs étaient requis quand il s'agissait d'assurer le succès des moissons, la bonne marche des saisons étant de la responsabilité des gouvernants (Motolinia 1970: 34-5; Anales de Cuauhtitlan 1949: 8). Pour les autres, c'étaient des enfants de prisonniers de guerre, ou, trop fréquemment, au point qu'à Texcoco il aurait fallu légiférer pour y mettre un frein, offerts ou vendus par leurs parents (Ixtlilxochitl 1: 405, 447). On achetait aussi des enfants à l'extérieur. L'immense majorité d'entre eux étaient offerts aux eaux et aux dieux de la pluie, qu'ils représentaient, peut-être en raison de leur aspect de nains, semblable à celui des Tlaloque. D'autres destinataires étaient Quetzalcoatl (Codex Vaticanus $A$ fol.20), qui, en tant que dieu du vent, appartient aux Tlaloque, un certain Aztacoatl (RG Tetela) et Tezcatlipoca, associé à la fête des enfants morts (Codex Magliabechiano fol. 36v) pour des raisons qui restent à éclaircir. Deux sources mentionnent des immolations d'enfants en cas de guerre (RG Coatepec et Chimalhuacan). Plusieurs, européennes ou assimilées, évoquent du sang d'enfants qui aurait été mélangé à des images de pâte ou à des cendres de divinités mais les textes nahuatl restent discrets sur ce point.

Parmi les guerriers captifs, certains pouvaient être choisis pour certains rites particuliers en fonction de leur nom personnel ou gentilice. Parmi les enfants, ceux qui avaient deux boucles au sommet de la tête étaient particulièrement recherchés (Sahagún II c.20), soit parce qu'elles évoquaient le tourbillon dans la lagune qui était un lieu de contact privilégié avec les Tlaloque, soit parce que le mot nahuatl pour les désigner signifiait aussi "grenier" et promettait l'abondance, ou encore, parce que ces enfants avaient un tonalli - un feu intérieur, une force vitale -, particulièrement puissant. ${ }^{16}$ Le cas des albinos, nains, bossus, contrefaits, macrocéphales, tous sacrifiables d'office, semble-t-il, est plus difficile (Tezozomoc 1878: 517, 563, 601; Sahagún VII c.1). Certains d'entre eux au moins étaient pourchassés, tous étaient mis à part, en particulier dans l'entourage du souverain, pour son divertissement mais surtout, sans doute, pour les contrôler, comme le porte à croire le fait que Montezuma II avait 
également concentré autour de lui, à Mexico, les fils des rois, les images de dieux et les animaux de l'empire. Ces personnes étaient immolées quand il y avait manque ou excès de pluie et lors d'éclipses du soleil, soit quand il y avait trop ou trop peu de soleil. Les nains et bossus devaient avoir des affinités avec les Tlaloque, les albinos étaient des élus du soleil. Enfin, dans le temple d'Iztaccinteotl, un dieu du maïs, on sacrifiait, paraît-il, des personnes qui souffraient de maladies contagieuses comme la gale ou la dartre, voire la lèpre, maladies attribuées à la déesse de l'amour Xochiquetzal (Torquemada 2:150-1).

Il est d'autres personnes de la cité qui, libres, pouvaient être appelées au sacrifice ou qui le choisissaient. On dit qu'au besoin, le roi pouvait faire sacrifier n'importe quel citoyen (Cortés, RG d'Atlatlahuaca...). Lors de la fête de la moisson (tlacaxipehualiztli), dans certaines régions, on sacrifiait un étranger de passage qui probablement représentait la dernière gerbe de maïs (RG Teotitlan). Pour la fête des montagnes, on sacrifiait deux vierges du lignage royal de Tezcacoatl (Durán 1:154), appelé ainsi semble-t-il d'après un des quatre guides des pérégrinations mexicas. On sait par recoupements qu'elles représentaient les déesses Ayopechtli et Atlacoaya, associées à l'eau et aux semis. Outre ces marginales du haut de la société, il y avait celles du bas. L'affirmation de Serna (1892:357-8) selon laquelle des jeunes femmes de petite vertu étaient recueillies par des prêtres qui leur promettaient de les établir mais les sacrifiaient au cours d'une fête est probablement une broderie sur la description que Sahagún (II c.30) fait de la fête d'ochpaniztli. Mais il y avait aussi les vraies prostituées, qui, en tepeilhuitl, fête au cours de laquelle on célébrait les amours, s'offraient librement au sacrifice en se maudissant et en injuriant les femmes honnêtes (Torquemada 2: 299). Leur mort est à rapprocher d'un passage de Sahagún (X c.15, 1950-81, 10:55) qui les caractérise comme menant une vie d'esclave baigné ou de victimes sacrificielle: peut-être vise-t-on leur mode de vie aussi luxueux et éphémère que celui des personnificateurs de dieux - d'autant plus que souvent, elles finissaient par se vendre comme esclaves. On trouvait aussi des volontaires parmi les musiciens, en échange de l'honneur de jouer du tambour lors d'une fête (Codex Tudela). 


\section{BIBLIOGRAPHIE}

ACOSTA, J. de, 1962 [1589] : Histoire naturelle et morale des Indes occidentales. Traduit par J. Rémy. Payot, Paris.

\section{Anales de Cuauhtitlan. Voir Codex Chimalpopoca} BERLIN, H. (éditeur)1948 : Anales de Tlatelolco (Unos Annales Históricos de la Nación Mexicana) y Códice de Tlatelolco. Traduit par $\mathrm{H}$. Berlin. Antigua Librería Robredo, Mexico.

BOSCH GARCÍA, C. 1944 : La esclavidud prehispánica entre los aztecas. Mexico.

BRODA, J. 1970: Tlacaxipehualiztli : A reconstruction of an Aztec calendar festival from 16th century sources. Revista Española de Antropología Americana 6:197-274.

CARMACK, R. M. 1976 : La estratificación quicheana prehispánica. In Estratificación social en la Mesoamérica prehispánica, édité par P. Carrasco and J. Broda, pp. 245277. INAH, Mexico.

CASO, A. 1953, El Pueblo del Sol. FCE, Mexico.

CASTAÑEDA PAGANINI, R. 1959, La cultura tolteca-pipil de Guatemala. Editorial del Ministerio de Educación Pública "José de Piñida Ibarra.", Guatemala.

CASTILlO, C. DEL 1991, Historia de la venida de los mexicanos y otros pueblos e Historia de la conquista. Traduit par F. Navarrete Linares. INAH, Mexico.

Cervantes de SAlazar, F. 1985, Crónica de la Nueva España. Porrúa, Mexico.

CHIMALPAHIN QUAUHTLEHUANITZIN, DOMINGO F. DE SAN ANTON MUÑON 1965, Relaciones originales de Chalco Amaquemecan. Traduit par S. Rendón.

FCE, Mexico. 1991, Memorial Breve acerca de la fundación de la ciudad de Culhuacan. Traduit par V. M. Castillo. UNAM, Mexico.

Clavijero, Fr. J. 1964, Historia antigua de México, edited by M. Cuevas. Porrúa, Mexico.

CODEX CHIMALPOPOCA

1938, Die Geschichte der Königreiche von Colhuacan und Mexico. Traduit et commenté parW. Lehmann. Quellenwerke zur alten Geschichte Amerikas 1, Kohlhammer, Stuttgart-Berlin.

1945, Códice Chimalpopoca. Anales de Cuauhtitlan y Leyenda de los Soles. Traduit par P. F. Velázquez. UNAM, Mexico. 
COMBÈS, I. 1992, La tragédie cannibale chez les Tupi-Guarani. PUF, Paris.

CORTES, H. 1963, Cartas y documentos. Porrúa, Mexico.

Díaz DEl CASTILlo, B. 1947, Verdadera historia de los sucesos de la conquista de la Nueva España. Biblioteca de Autores Españoles 26, Ediciones Atlas, Madrid.

DURAN, F. D. 1967, Historia de los indios de la Nueva España e Islas de la Tierra Firme. Edité par A. M. Garibay K. 2 vols., Porrúa, Mexico.

GARIBAY K., A. M. 1964-1968, Poesia nahuatl, 3 vols.UNAM-IIH, Mexico.

GIRARD, R. 1972 La violence et le sacré. Grasset, Paris.

GOMARA, FR. LOPEZ de, 1965-1966, Historia general de las Indias. 2 vols., Iberia, Barcelone.

GRAULICH, M.

1987, Mythes et rituels du Mexique ancien préhispanique. Académie Royale de Belgique, Mémoires de la Classe des Lettres 67,3, Palais des Académies, Bruxelles.

1991, L'inauguration du temple principal de Mexico en 1487. Revista Española de Antropología Americana 21:121-143.

1992, The Aztec "Templo Mayor" Revisited. Ancient America. Contributions to New World Archaeology. Oxbow Monograph 24: 19-32.

1997, Chasse et sacrifice humain chez les Aztèques. Académie Royale des Sciences d'Outre-Mer, Bulletin des Séances 43, 4: 433-46.

1999 Ritos aztecas: las fiestas de las veintenas. Instituto Nacional Indigenista, Mexico.

2000a, Tlahuicole, un héroe tlaxcalteca controvertido. In El héroe entre el mito y la historia, édité par F. Navarrete and G. Olivier, pp. 89-99. UNAM, CEMCA, Mexico.

2000b, Coyolxauhqui y las mujeres desnudas de Tlatelolco. Estudios de Cultura Nahuatl $31: 77-94$

HASSIG, R. 1988, Aztec Warfare: Imperial Expansion and Political Control. University of Oklahoma Press, Norman-London.

HELFRICH, K. 1973, Menschenopfer und Tötungsrituale im Kult der Maya. Monumenta Americana 9, Gebr.Mann, Berlin.

JONGHE, E. de (editor) 1905, Histoyre du Méchique. Journal de la Société des Américanistes de Paris 2,1:10-44.

IXTLILXÓCHITL, F. de ALVA, 1975-1977, Obras históricas, édité par E. O'Gorman. 2 vols., UNAM, Mexico. 
KLEIN, C. 1994, Fighting with feminity: gender and war in Aztec Mexico. Estudios de Cultura Náhuatl 24: 219-253.

LANDA, D. de , 1941, Landa's Relación de las cosas de Yucatan. Traduit et annoté par A. M. Tozzer. Papers of the Peabody Museum of American Archaeology and Ethnology, Harvard University XVIII, Cambridge.

LAS CASAS, FRAY B. de,

1967, Apologética historia sumaria, édité par E. O'Gorman. 2 vols., UNAM, Mexico.

1985, Obra indigenista, édité par J. Alcina Franch, Alianza, Madrid.

LOPEZ Austin, A. 1965, El Templo Mayor de Mexico-Tenochtitlan según los informantes indígenas. Estudios de Cultura Náhuatl 5: 75-102.

MARTINez CORTES, F. 1974, Pegamentos, gomas y resinas en el México prehispánico. SepSetentas, Mexico.

MARTIR De ANGleria, P. 1964-1965, Décadas del Nuevo Mundo. 2 vols., J. Porrúa, Mexico.

MENDIETA, F. J. de, 1945, Historia eclesiástica Indiana. 4 vols., Chávez Hayhoe, Mexico.

METRAuX, A. 1967, Religions et magies indiennes d'Amérique du Sud. Gallimard, Paris.

MOLINA, F. A. de, 1970, Vocabulario en lengua castellana y mexicana y mexicana y castellana. Porrúa, Mexico.

MOTOliniA, F. T. de BENAVENTE, 1970, Memoriales e Historia de los Indios de la Nueva España, édité par F. de Lejarza. Ediciones Atlas, Madrid.

MUÑoz Camargo, D. 1892, Historia de Tlaxcala. Secretaría de Fomento, Mexico.

GUILHEM, O. 1997, Moqueries et métamorphoses d'un dieu aztèque. Tezcatlipoca, le "Seigneur au miroir fumant". Mémoires de l'Institut d'Ethnologie XXXIII, Musée de l'Homme, Paris.

OVIEDO Y VALDES, G. FERNANDEZ de, 1959, Historia general y natural de las Indias. 5 vols., Biblioteca de Autores Españoles, Atlas, Madrid.

PIHO, V.

1970, Función y simbolismo del atavío azteca. Actes du 38e Congrès International des Américanistes, Stuttgart 1968, (2):377-384. 
1974, La jerarquía militar azteca. Actes du 40e Congrès International des Américanistes, Rome-Gênes 1972, (2) :273-288.

POMAR, J. B. 1986, Relación de la ciudad y provincia de Tezcoco. Relaciones geográficas del siglo XVI: México, édité par R. Acuña, t. 3, UNAM, Mexico.

PREUSS , K. TH.

1903a, Die Feuergötter als Ausgangspunkt zum Verständnis der mexikanischen Religion in ihrem Zusammenhange. Mitteilungen der Wiener Anthropologischen Gesellschaft 33: 129-233.

1903b, Die Sünde in der Mexikanischen religion. Globus 83: 253-7, 268-73.

1984-1988, Relaciones geográficas del siglo XVI, édité par R. Acuña. 10 vols., UNAM, Mexico.

SAHAGUN, B. de

1956, Historia general de las cosas de Nueva España, édité par A.M. Garibay K. Porrúa, Mexico.

1958, Ritos, Sacerdotes y Atavíos de los Dioses. Traduit par M. León-Portilla. UNAM, Mexico.

1950-1981[1577] Florentine Codex, General History of the Things of New Spain. Translated by A. J. O. Anderson and Ch. E. Dibble. 12 vols. The School of American Research and the University of Utah, Santa Fe, New Mexico.

SELER, E.

1890, Das Tonalamatl der Aubinschen Sammlung und die Verwandten Kalendarbücher. Actes du $7^{\mathrm{C}}$ Congrès International des Américanistes, Berlin $1888: 521-735$.

1902-1923, Gesammelte Abhandlungen zur Amerikanischen Sprach-und Altertumskunde. 5 vols., Asher, Berlin.

SERNA, J. de la, 1892, Manual de ministros de indios para el conocimiento de sus idolatrías y extirpación de ellas. Anales del Museo Nacional de México 6: 261-480.

SOUSTELLE, J.

1940, La pensée cosmologique des anciens Mexicains (Representations du monde et de l'espace). Hermanet, Paris.

1955, La vie quotidienne des Aztèques à la veille de la conquête espagnole. Hachette, Paris.

SPRANZ, B.

1964, Göttergestalten in den mexikanischen Bilderhandschriften der Codex Borgia Gruppe. Acta Humboldtiana 4. F. Steiner, Wiesbaden.

SULLIVAN, T. D. 1963, Nahuatl proverbs, conundrums, and metaphors, collected by Sahagún. Estudios de Cultura Náhuatl 4:93-177. 
TEZOZOMOC, F. ALVARADO

1878, Crónica mexicana... precedida del Códice Ramírez. Ireneo Paz, Mexico.

1949, Crónica mexicáyotl. Traduit et établi par A. León. UNAM, Mexico.

TORQuemada, F. J. de, 1969, Monarquía Indiana. 3 vols., Porrúa, Mexico.

TOVAR, J. de, 1972, Manuscrit Tovar. Origines et croyances des Indiens du Mexique, édité par J. Lafaye. Akademische Druck- und Verlagsanstalt, Graz.

TOZZER 1941, voir Landa 1941. 


\section{NOTES}

\footnotetext{
1 Voir aussi Ixtlilxochitl, Historia chichimeca c.38, 1975-77: 2: 103. Pour lui, c'est la Triple Alliance qui se réunit en conseil de guerre "con sus capitanes y consejeros".

${ }^{2}$ Sur cette guerre et ses autres motivations, notamment politiques, voir Graulich 1994; Durán 1967, 2: 232-3, 235, 417; 1: 32-3; Muñoz Camargo 1892: 116 ; Motolinia 1970: 219-20.

${ }^{3}$ Ainsi, le premier recevait le corps et la cuisse droite, le second la cuisse gauche, le troisième le bras droit, le quatrième bras gauche, le cinquième l'avant-bras droit et le sixième l'avant-bras gauche.
}

${ }^{4}$ Pour Cervantes de Salazar, qui diffère ici, celui qui a fait 5 prisonniers "mudava el traje del cortar del cabello, hazia las orejas, hechas dos rasuras : a este llamaban quachic qu'es el titulo mas honroso. Auiendo muerto seis, se podia cortar el cabello de la media cabeça hasta la frente"; avec 7 tués, il devient cuauhnochtli, avec 10 , tlacatecuhtli et en outre seigneur d'un village où il se repose pour le restant de ses jours. Voir sur ce sujet Piho 1970, 1974; Hassig 1988. A Tlaxcala, il y avait des vaillants qui avaient pris ou tué plus de 20,90 et même 100 personnes: Motolinia 1970: 42.

5 Le sacrifice de guerriers est en effet double: l'excision du coeur pour le soleil, la décapitation subséquente pour la terre.

${ }^{6}$ La source ne mentionne pas la Terre, second destinataire, mais celle-ci était présente en plusieurs endroits et sous plusieurs formes dans la pyramide principale de Mexico.

7 "... and here, to Mexico, from everywhere, were brought captives, called "tribute-captives" (All who lay surrounding, those who held the enemy borders, those who dwelt on the enemy borders, brought their captives, their prisoners here. [These] became their tribute-captives [maltequihoan]. The stewards [calpixque'], each of the stewards, guarded them here.... and if one of them fled,... they replaced him; a man was purchased; they delegated another [qujxiptlaiotiaia], they placed in his stead. Then the slaves died, when the feast day was celebrated.

${ }^{8}$ Les Anales de Cuauhtitlan p. 3 énumèrent comme buts des Chichimèques qui se dispersent en 1 Silex 804, avant Quetzalcoatl, toutes les régions qui resteront insoumises à l'empire aztèque (mais sans préciser ce point): Michoacan, Cohuixcon Yopitzinco, Totollan (un quartier de Tlaxcala), Tepeyacac (près de Quecholac), Quauhquechollan, Huexotzinco, Tlaxcala, Tliliuhquitepec, Zacatlantonco et Tototepec.

${ }^{9}$ Sur ce sujet, voir Klein 1994; Graulich 2000b

${ }^{10}$ Dans les codex Telleriano-Remensis fol. 32v, 38v, 39r, 40 r, 40v, 41r, 42v, les guerriers sacrifiés à Mexico lors des occasions les plus diverses en sont ornés. Voir aussi, pex, certains objets archéologiques comme l'os gravé du Musée de l'Homme, Seler 1902-23, 2: 682.

${ }^{11}$ Cristóbal del Castillo, auteur assez tardif, doit toutefois être manié avec prudence vu sa manie de broder. Le discours de la divinité à Huitzilopochtli évoque assez fort celui de Dieu à Moïse avant l'arrivée dans la Terre promise (Exode, Lévitique, Nombres, Deutéronome).

${ }^{12}$ Voir aussi Garibay, Poesía Náhuatl 2: 5-6, 42-3, 53-4; Romances de los señores de la Nueva España f.42 $\mathrm{v}^{\circ}$; l'hymne à Mère des Dieux, Teteo innan, recueilli par Sahagún 1956, 4: 296; à rapprocher du livre 2, App., 1956, 1: 246-7. On envoie de la craie et des plumes comme déclaration guerre: Broda 1970: 204.

${ }^{13}$ Mendieta 1:110: les gens mettent dans les champs des pierres teintes de chaux ou de craie. 
${ }^{14}$ Dans Sahagún (CF 2: 66), il est dit qu'on prenait l'ixiptla parmi les captifs et que "là on choisissait quelqu'un s'il paraissait convenir, s'il était bien de corps. Alors on le prenait et les [sic] confiait aux intendants. Mais celui destiné à être esclave, le capteur le tue". Je ne sais que faire de cette dernière phrase. Les esclaves sacrificiels incarnant des dieux devaient eux aussi être bien faits physiquement et purs.

${ }^{15}$ Cervantes de Salazar 1: 46; Tezozomoc 1878: 321; Mendieta 1: 144; Tovar 228; Pomar 1986: 89; Gómara 1975-7: 95...

${ }^{16}$ Le tonalli des victimes renforce celui des dieux ou des rois. Si je l'invoque ici à propos de ces enfants à tourbillons, c'est grâce à Michel Duquesnoy, à qui je dois la précieuse information qui suit et que je remercie vivement. Il a donc entendu dire par Horacio, un de ses principaux informateurs à San Miguel Tzinacapan, que "el tonal es el remolino que tiene uno en la cabeza. es el remolino de cabellos. Es mejor tener dos remolinos porque es fuerte de su tonaltsi $[. . . .$.$] Porque este remolino$ que tenemos es como un enchufa que tenemos con el sol (in tonal), por allí entra el calor (in tona) del sol. Cuando tiene uno dos remolinos, tiene dos soles, es como un favor del sol. Pero es muy dañino para las cosas." 